\section{Preconditioning Techniques in the Analysis of Finite Metamaterial Slabs}

\author{
Eduard Ubeda, Juan M. Rius, and Jordi Romeu
}

\begin{abstract}
In the method of moments (MoM) electric field integral equation (EFIE) analysis of slabs of metamaterials, we show that a left-preconditioning scheme by blocks gathering interactions between basis functions belonging to each basic cell of the metamaterial reaches convergence in less iterations and with less computational time than a left-preconditioner based on discarding interactions between basis functions beyond a given distance.
\end{abstract}

Index Terms-Electromagnetic scattering, method of moments (MoM), numerical analysis, preconditioning.

\section{INTRODUCTION}

Metamaterials are composite arrays of conducting elements-thin strips and elementary spires-which, at resonance, behave as materials with negative index of refraction [1]. Since these structures have large electrical dimensions, they can be modeled as infinite and periodic, which allows the method of moments (MoM) analysis based on the expansion in the transformed domain (formulation in Floquet modes) [2]. The required memory and CPU time are moderate since they only depend on the discretization of one single cell. However, this approach is too restrictive for a general real-life case because the effect of the borders is neglected and because a periodic excitation needs to be assumed.

A MoM-formulation based on the discretization of the whole structure is a brute-force method that leads to the accurate solution for a realistic case under an arbitrary exciting source. Due to the modeling of the elements in the array as open surfaces, the MoM-EFIE formulation needs to be employed. Since metamaterials are resonant and finely meshed to allow for the intricate details of the split ring resonators (SRRs), the MoM-EFIE formulation results in poorly-conditioned matrices and thus the iterative algorithm converges very slowly or even stagnates. It is thus obligatory the implementation of robust iterative methods [3] together with efficient preconditioning schemes [3], [4] to ensure fast convergence. In this work, the generalized minimum residual (GMRES) method is adopted as iterative algorithm because it is more robust to poorly-conditioned systems than other Krylov-subspace algorithms, such as the generalized conjugate residual (GCR) algorithm. Also, for electrically large arrays, which manage a large number of unknowns, the implementation of the multilevel fast multipole algorithm (MLFMA) [5] accelerates the matrix-vector product at each iterative step.

\section{THEORY}

The preconditioning techniques are based on the generation of a matrix, the preconditioner, which pre-multiplies both sides of the original linear system of equations with the objective of reducing the condition

Manuscript received July 15, 2004; revised September 14, 2005. This work was supported by the "Departament d'Universitats, Recerca i Societat de la Informació (DURSI)" under "Distinció de la Generalitat de Catalunya per a la Promoció de la Recerca Universitaria" and the Spanish "Comisión Interministerial de Ciencia y Tecnología (CICYT)" through the "Ramón y Cajal" Programme and the Grants TIC 2001-2364-C01-01, TIC 2003-09317-C03-03.

The authors are with the Signal Theory and Communications Department, Universitat Politècnica de Catalunya, 08034 Barcelona, Spain (e-mail: ubeda@tsc.upc.edu).

Digital Object Identifier 10.1109/TAP.2005.861508 number of the associated matrix and, therefore, the number of iterations to reach convergence. The preconditioner $P$ must be a good, computationally cheap and sufficiently sparse approximation of inv $(Z)$, where $Z$ denotes the linear system matrix arising out of the MoM formulation: $Z x=b$.

In this paper, we use left-preconditioned schemes [3] so that in the GMRES-search of the solution the residual norm of the system $P Z x=$ $P b$ is minimized. The construction of $P$ comprises the generation of a sparse matrix $M$ including relevant interactions in $Z$ and the computation of $P$ through an approximation of $\operatorname{inv}(M)$ because the direct computation of $\operatorname{inv}(M)$ increases the memory requirements dramatically. The incomplete lower upper (ILU) decomposition or memory-efficient related implementations such as ILU(0) and ILUT [3] are employed to keep the matrix element fill-ins restricted. The ILU decomposition is computed in the same column-oriented manner as the LU factorization but, during the process, all the entries in each column of either $\mathrm{L}$ or $\mathrm{U}$ below a preset threshold are discarded. This threshold is defined by the parameter drop-tolerance (drop-tol). ILU-based preconditioning techniques [6], [7] are widely used and become normally more efficient than the approximate inverse preconditioners (AIPC) [3], which are based on the more time-consuming minimization of the Frobenius-norm of the residual matrix $I-Z P$. Recently, Eibert [8] has proposed a preconditioning scheme that implicitly accounts for $\operatorname{inv}(M)$ through an approximate iterative search of $P$ nested in the GMRES-search of the solution. It excels as a very memory-efficient scheme, especially suited for electrically large objects. We obtain the two preconditioning schemes under study in this work through the ILU decomposition of $M$. These two preconditioners differ in the way $M$ is constructed.

\section{A. Geometric Banded-Diagonal (Band-Geom)}

In the generation of $M$ those interactions between pairs of basis functions within a given distance-the radius of preconditioning (Rpc) - are considered. For a given testing function, a row in $Z$, we take into account the MoM-interactions with all the basis functions belonging to a sphere with radius $R p c$ and centered at that testing function. The selection of the relevant elements in $Z$ for $M$ is thus carried out in terms of their physical proximity, which represents a conventional strategy to define the band around the main-diagonal of $M$ [6], [7], [9]. Other preconditioners [8] select the band in $M$ by keeping the matrix elements with largest modulus, which, in our experience, becomes somewhat less effective.

\section{B. Geometric Block-Diagonal (Block-Geom)}

In accordance with the conventional definition of the block-diagonal preconditioner, $M$ is defined from the extraction of a set of square blocks along the main diagonal from the original matrix $Z$ [4]. Since the ILU of a block-diagonal matrix is the summation of the ILU of each of the diagonal-blocks, the computational requirements are linked with the required memory and CPU time to handle each block separately. This allows the management of electrically large problems where the banded-diagonal schemes fail because their memory requirements are beyond the available resources.

The block-diagonal approach is very often refined so that each block gathers all the interactions between the elements belonging to a limited region of the geometry [5], [10]. J. Song [5] propose a block-diagonal scheme by assigning the blocks to the interactions in the cubes at the finest level of the MLFMA-MoM-CFIE formulation. However, since the MoM-CFIE formulation is better conditioned than MoM-EFIE [6], insight into the choice of the preconditioner is less peremptory than 


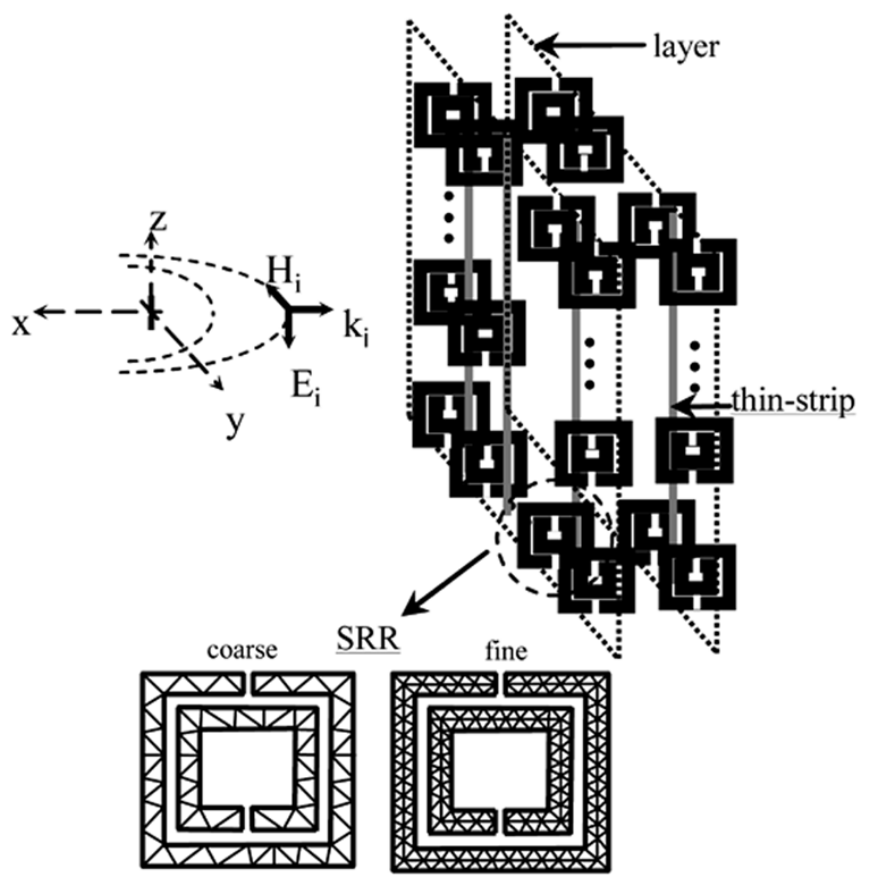

Fig. 1. Finite composite array with an exciting elementary dipole at a distance of $55.5 \mathrm{~mm}$. The SSRs are meshed with either a coarse or a fine discretization.

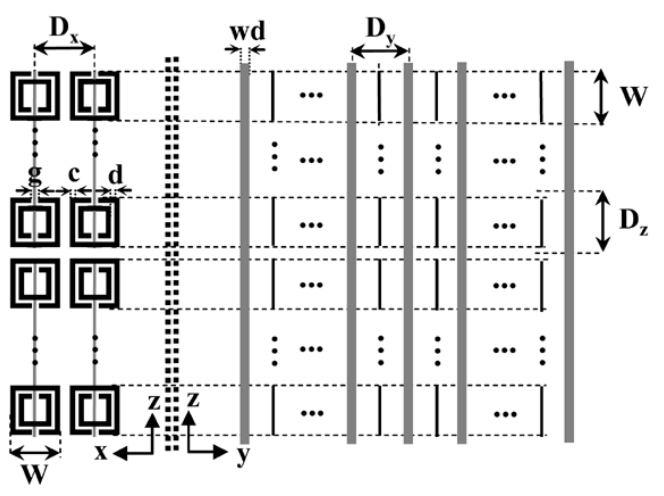

Fig. 2. $X Z$ and $Y Z$ sections of the 2 layer array of SRR and thin-strips: $D_{x}=$ $8 \mathrm{~mm}, D_{y}=8 \mathrm{~mm}, D_{z}=10 \mathrm{~mm}, W=6.6 \mathrm{~mm}, w d=0.5 \mathrm{~mm} ; g=0.3 \mathrm{~mm}$; $d=0.2 \mathrm{~mm} ; c=0.8 \mathrm{~mm}$.

in the MoM-EFIE analysis. J. R. Poirier [10] show the good performance of a block-diagonal scheme with the transpose-free quasi-minimal residual method (TFQMR) iterative algorithm in the MoM-EFIE analysis of patch-arrays. In view of the array structure of the metamaterials, we propose to assign each of the blocks in $M$ to the interactions between the elements inside each of the basic cells of the metamaterial; that is, the SRRs and the thin-strips.

\section{GeOMETRIC AND COMPUTATIONAL CONSIDERATIONS}

We analyze composite arrays of split ring resonators (SRRs) regularly distributed over the $z y$ plane and $z$-oriented thin-strips (see Figs. 1 and 2). The SRRs are formed by two concentric slotted square rings [11] oriented perpendicularly to the $y z$ plane whereas the thin-strips lie onto the $y z$ plane. In this paper, we name an arbitrary composite array with $m$ layers over the $x$-direction of a combined structure of $n x n$ SSRs and $p z$-oriented thin-strips as [ $m$ layer, nxn SRR, $p$ thin-strips].
At the working frequency $(5.4 \mathrm{GHz})$, where our metamaterial slab is resonant, the same structure with only SSRs shows very little transmitted power and behaves as magnetic conductor [12]. We have placed an exciting elementary dipole in front of the composite structure as shown in Fig. 1. Note that the dipole is oriented so that the radiated electric and magnetic fields are parallel, respectively, to the thin-strips and to the axes of the SSRs in order to enhance the electromagnetic coupling on the structure.

The composite arrays have been analyzed with a Rao-WiltonGlisson (RWG) MoM-EFIE formulation [13] with accurate integration of the Kernel. In the self-interactions, the integration of $1 / \mathrm{R}$ is carried out analytically [14] and the field-integration is computed folllowing a 3-point Gaussian quadrature rule. For interactions between different triangles, the integration over the source triangle is undertaken numerically with a 4-point rule and the field-integration is carried out with 1 point at the centroid. Since this is a problem with four-folded symmetry, we have only computed one fourth of the MoM-interactions to save time and memory in the generation of $Z$ and in the matrix-vector products. We have used a processor AMD Athlon(tm) XP 1800+ (1.54 $\mathrm{GHz}$ ) and 1.50 Gbyte of RAM.

The meshing of the SSRs has been made with either a coarse or a fine grid, which involve, respectively, one or two rows of triangles across the transversal section (see Fig. 1). Such meshings represent an average length of the sides of the facets of, respectively, $0.02 \lambda$ and $0.01 \lambda$. In all the cases, we have stopped the iterative solver GMRES for relative residual norms below $0.1 \%$. To check the behavior of bandgeom and block-geom, we have analyzed two sets of composite arrays (moderarely small and moderately large).

\section{A. Moderately Small}

This set of arrays follows the structure depicted in Fig. 2, which yields the composite arrays [ $m$ layer, $n x n S R R, n+1$ thin-strips]. The thin-strips are continuous along $z$ and discretized with a mesh-size of $0.02 \lambda$. We have tested the cases $m=1,2$ and $n=6,8$. The matrices related with the preconditioning scheme $M$ and $P$ could be saved in memory whereas $Z$ had to be stored in disk.

\section{B. Moderately Large}

This set of composite arrays also follows the general structure depicted in Fig. 2 but the thin-strips are somewhat wider ( $w d=1.5 \mathrm{~mm}$ ) and noncontinuous along $z$. They are split in portions of length 27.52 $\mathrm{mm}$ (about half a wavelength at $5.4 \mathrm{GHz}$ ) so that they can be more easily manufactured. We have tested the composite arrays [1 layer, $18 \times 16$ SRR, 15 thin-strips] and [2 layer, $18 \times 16$ SRR, 15 thin-strips], which lead to moderately big electrical dimensions $(3.18 \lambda \times 2.26 \lambda)$. Since the conventional MoM approach requires too much computational effort, we use the MLFMA instead. In general, some loss of accuracy must be presumed but with an adequate value for the precision parameter, this error becomes unnoticeable for far-field magnitudes. In our experience, a precision factor of 2, within the range proposed by J. Song in [4], is satisfactory. Moreover, we have adopted a minimum box size of $0.1 \lambda$ and an interlevel interpolation degree of 4 . We also relax the meshing criterion in the thin-strips to mesh-sizes of $0.04 \lambda$. Due to the large number of unknowns to be handled, the matrices $M$ and $P$ were to be stored in disk along with the near-interactions linked to MLFMA.

\section{RESUlts}

In order to establish a fair comparison between both preconditioners, we have checked first the best-performing configurations. Band-geom reduces most the number of iterative steps in least time for $R p c=7 \mathrm{~mm}$. 
TABLE I

Times AND Number of ITERATIVE STEPS TO REACH CONVERGENCE For THE Moderately SMALL COMPOSITE ARRAYS WITH 1 OR 2 LAYERS AND COARSE DISCRETIZATION

\begin{tabular}{|c|c|c|c|c|c|}
\hline \multirow{5}{*}{ Geom } & mesh & \multicolumn{4}{|c|}{ Coarse } \\
\hline & Layers & \multicolumn{2}{|c|}{1} & \multicolumn{2}{|c|}{2} \\
\hline & SRR & $6 \times 6$ & $8 \times 8$ & $6 \times 6$ & $8 \times 8$ \\
\hline & strips & 7 & 9 & 7 & 9 \\
\hline & $\mathrm{Ne}$ & 3932 & 6808 & 7708 & 13616 \\
\hline \multirow{2}{*}{$\mathrm{Tm}$} & Band & 2.2 & 4.9 & 6.2 & 20.5 \\
\hline & Block & 1.6 & 3.2 & 3.6 & 8.6 \\
\hline \multirow{2}{*}{$\mathrm{Tp}$} & Band & 0.7 & 1.3 & 3.5 & 14.2 \\
\hline & Block & 2.3 & 3.1 & 4.6 & 5.4 \\
\hline \multirow{3}{*}{ Ttotal } & Band & 8.0 & 38.2 & 48.2 & 253.1 \\
\hline & Block & 7.6 & 33.6 & 39.4 & 202.6 \\
\hline & No prec & 1672.1 & 16037.2 & 28827.5 & 203257.3 \\
\hline \multirow{3}{*}{ steps } & Band & 15 & 35 & 32 & 61 \\
\hline & Block & 12 & 29 & 27 & 50 \\
\hline & No prec & 418 & 725 & 835 & 1359 \\
\hline
\end{tabular}

TABLE II

Times AND Number of ITERATIVE STEPS TO REACH CONVERGENCE For THE Moderately SMall COMPOSITE ARRAYS WiTH 1 OR 2 LAYERS AND FINE DISCRETIZATION

\begin{tabular}{|c|c|c|c|c|c|}
\hline \multirow{4}{*}{ Geom } & mesh & \multicolumn{4}{|c|}{ Fine } \\
\cline { 2 - 6 } & Layers & \multicolumn{2}{|c|}{1} & \multicolumn{2}{|c|}{2} \\
\cline { 2 - 6 } & SRR & $6 \times 6$ & $8 \times 8$ & $6 \times 6$ & $8 \times 8$ \\
\cline { 2 - 6 } & strips & 7 & 9 & 7 & 9 \\
\cline { 2 - 6 } & Ne & 15848 & 24856 & 31696 & 49712 \\
\hline \multirow{4}{*}{ Tm } & Band & 40.3 & 85.9 & 141.8 & 720.2 \\
\cline { 2 - 7 } & Block & 15.4 & 24.2 & 31.5 & 76.0 \\
\hline \multirow{4}{*}{ Tp } & Band & 18.3 & 21.8 & 245.7 & 640.2 \\
\cline { 2 - 7 } & Block & 15.1 & 21.3 & 23.7 & 39.2 \\
\hline \multirow{5}{*}{ Ttotal } & Band & 148.5 & 473.8 & 2607.0 & 9073.8 \\
\cline { 2 - 7 } & Block & 104.3 & $\mathbf{4 0 0 . 4}$ & $\mathbf{1 6 3 4 . 3}$ & $\mathbf{6 4 1 8 . 2}$ \\
\cline { 2 - 7 } & No prec & 44188.9 & \multicolumn{2}{|c|}{ Extremely slow convergence } \\
\hline \multirow{3}{*}{ Steps } & Band & 19 & 32 & 35 & 67 \\
\cline { 2 - 6 } & Block & $\mathbf{1 6}$ & $\mathbf{3 1}$ & $\mathbf{2 5}$ & $\mathbf{5 5}$ \\
\cline { 2 - 6 } & No prec & 910 & \multicolumn{3}{|c|}{ Extremely slow convergence } \\
\hline
\end{tabular}

The best-performing drop-tolerances for band-geom and block-geom turn out to be, respectively, of $4 \mathrm{e}-5$ and $1 \mathrm{e}-5$. Since the resonance of the whole geometry is based on the resonance of each SRR separately [15], it is reasonable that the best-performing preconditioning configurations prevail as the electrical dimensions of the arrays increase. Note that the optimum radius of preconditioning $(7 \mathrm{~mm})$ is very close to the spatial periodicities of the arrays (see Fig. 2), which are very small compared with the dimensions of the whole geometry. This is very advantageous because we can spare many memory resources in the construction of the matrix $M$. Indeed, in the MoM-MLFMA analysis of these moderately large composite arrays, instead of constructing $M$ with the whole nearfield MLFMA matrix, as suggested in [6] [7] for a wide variety of large problems, it is sufficient to establish regions with dimensions restricted to roughly $\lambda / 4$.

In Tables I, II and III, we display the performance of the optimum configurations of band-geom and block-geom for all the composite arrays, [1 layer, $6 \times 6 S R R$, 7 thin-strips], [2 layer, $6 \times 6 S R R$, 9 thinstrips], [1 layer, $8 \times 8$ SRR, 9 thin-strips], [2 layer, $8 \times 8$ SRR, 9 thinstrips], [1 layer, $18 \times 16 \mathrm{SRR}$, 15 thin-strips] and [2 layer, $18 \times 16 \mathrm{SRR}$,
TABLE III

Times AND Number of ITERATIVE STEPS TO REACH CONVERGENCE FoR THE MODERATELY LARGE COMPOSITE ARRAYS WITH 1 OR 2 LAYERS AND COARSE OR FINE DISCRETIZATION

\begin{tabular}{|c|c|c|c|c|c|}
\hline \multirow{5}{*}{ Geom } & SRR & \multicolumn{4}{|c|}{$18 \times 16$} \\
\hline & Strips & \multicolumn{4}{|c|}{15} \\
\hline & Mesh & \multicolumn{2}{|c|}{ Coarse } & \multicolumn{2}{|c|}{ Fine } \\
\hline & Layers & 1 & 2 & 1 & 2 \\
\hline & $\mathrm{Ne}$ & 21120 & 47424 & 104928 & 209856 \\
\hline \multirow{2}{*}{$\mathrm{Tm}$} & Band & 32.1 & 131.8 & 986.2 & 3514.4 \\
\hline & Block & 2.1 & 49.1 & 106.6 & 386.2 \\
\hline \multirow{2}{*}{$\mathrm{Tp}$} & Band & 3.9 & 40.2 & 108.2 & $\begin{array}{l}\text { Memory } \\
\text { overflow }\end{array}$ \\
\hline & Block & 15.8 & 38.9 & 140.2 & 377.6 \\
\hline \multirow{3}{*}{ Ttotal } & Band & 338.9 & 2910.5 & 14825.1 & $\mathrm{X}$ \\
\hline & Block & 333.7 & 2337.7 & 11307.6 & 92091.9 \\
\hline & No prec & \multicolumn{4}{|c|}{ Extremely slow convergence } \\
\hline \multirow{3}{*}{ Steps } & Band & 68 & 163 & 106 & $\bar{X}$ \\
\hline & Block & 66 & 137 & 84 & 208 \\
\hline & No prec & \multicolumn{4}{|c|}{ Extremely slow convergence } \\
\hline
\end{tabular}

15 thin-strips]. In Tables I and II, we show the results for the moderately small arrays, whereas in Table III we show the results for the moderately large arrays. $T m$ and $T p$ denote the times required to compute $M$ and $P$ and Ttotal denotes the total time, including $T p, T m$, and the GMRES-search time to reach convergence. In view of these tables, block-geom excels as best-performing for each composite array because it reaches convergence in less steps and less total computational time than band-geom. We display also the speed of convergence of the GMRES-search without preconditioning, which becomes for all the cases, as expected, much slower than with any of our two preconditioners. We have stopped the GMRES-search when the number of steps to reach a relative residual norm below $1 \%$ is above 1000 , which stands for an "extremely slow" speed of convergence.

From the observation of the values of $T p$ and $T m$, we see that when the size of the nonzero entries in the matrix $M$ is high (the composite array is either finely meshed or electrically big), the choice of block-geom is critical respect to band-geom. In these cases, the growth of $T \mathrm{~m}$ in band-geom is more abrupt because a specific search of the wanted interactions for each element over the whole geometry needs to be carried out. Also, the growth of $T p$ in block-geom is more moderate because the ILU decomposition is applied separately to each of the blocks in $M$

Finally, band-geom fails in solving [2 layer, $18 \times 16$ SRR, 15 thinstrips] and fine meshing (see Table III) because so many unknowns $(\mathrm{Ne}=209856)$ need to be handled that the computation of $P$ cannot be completed. Recently, Heldring [16] have introduced a preconditioning scheme that carries out the band-geom scheme by blocks. This scheme has allowed to solve a reflector antenna (with over half a million unknowns) on a Desktop PC. This preconditioner makes a systematic geometric rearrangement of the basis functions according to a preset number of blocks $(n b)$. Strictly speaking it is not a pure block-diagonal scheme because off-diagonal blocks still remain in $M$ and overload the ILU decomposition of $M$. However, this burden in the memory-management for the computation of $P$ can be partially kept under control by means of another drop-tolerance $\left(\right.$ drop $\left.-t_{o l}\right)$. In the analysis of the demanding case [2 layer, $18 \times 16$ SRR, 15 thin-strips] with fine meshing, a very good configuration of this preconditioner $(n b=$ $40 ; \mathrm{drop}-\mathrm{tol}=\mathrm{drop}-\mathrm{tol}_{2}=1 e-5 ; R p c=7 \mathrm{~mm}$ ) leads to 165603 sec of total computational time and 303 iterative steps. As shown in Table III, block-geom offers a $44.4 \%$ reduction of the total time. 


\section{CONCLUSION}

The preconditioning scheme adopting the interactions between elements inside a cell of the array —SSRs or thin-strips - as blocks excels as a suitable tool to analyze systematically and most efficiently finite composite structures in metamaterials. It has been compared with traditionally successful tools in the MoM-EFIE analysis such as the ILU preconditioner relying on a geometrically based selection of a bandeddiagonal portion of $Z$, for the cases where the required resources are available in our PC, and a blockwise memory-efficient modification for the case of problems with very large number of unknowns. In all the cases tested, the geometric block-diagonal preconditioner reaches convergence in less number of iterations and total computational time.

\section{REFERENCES}

[1] V. G. Veselago, "The electrodynamics of substances with simultaneously negative values of $\varepsilon$ and $\mu$," Sov. Phys. Usp., vol. 10, no. 509, 1968.

[2] C. C. Chen, "Scattering by a two-dimensional periodic array of conducting plates," IEEE Trans. Antennas Propag., vol. AP-18, pp. 660-665, Sep. 1970.

[3] Y. Saad, Iterative Methods for Sparse Linear Systems. Boston, MA: PWS, 1996.

[4] K. Forsman, W. Gropp, L. Kettunen, D. Levine, and J. Salonen, "Solution of dense systems of linear equations arising from integral-equation formulations," IEEE Antennas Propag. Mag, vol. 37, pp. 96-100, 1995.

[5] J. Song, C. C. Lu, and W. C. Chew, "Multilevel fast multipole algorithm for electromagnetic scattering by large complex objects," IEEE Trans. Antennas Propag., vol. 45, pp. 1488-1493, 1997.

[6] K. Sertel and J. L. Volakis, "Incomplete LU preconditioning for FMM implementation," Microw. Opt. Technol. Lett., vol. 26, no. 4, Aug. 2000.

[7] J. Lee, J. Zhang, and C-C. Lu, "Incomplete LU preconditioning for large scale dense complex linear systems from electromagnetic wave scattering problems," J. Comput. Phys., vol. 185, pp. 158-175, Feb. 2003.

[8] T. F. Eibert, "Iterative near-zone preconditioning of iterative method of moments electric field equation solutions," IEEE Antennas Wireless Propag. Lett., vol. 2, pp. 101-102, 2003.

[9] M. Carr, M. Bleszynski, and J. L. Volakis, "A near-field preconditioner and its performance in conjunction with the BiCGsatbn(ell) solver," IEEE Antennas Propag. Mag., vol. 46, no. 2, pp. 23-30, Apr. 2004.

[10] J. R. Poirier, P. Borderies, R. Mittra, and V. Varadarajan, "Numerically efficient solution of dense linear system of equations arising in a class of electromagnetic scattering problems," IEEE Trans. Antennas Propag., vol. 46, no. 8, pp. 1169-1175, Aug. 1998.

[11] R. A. Shelby, D. R. Smith, S. C. Nemat-Nasser, and S. Schultz, "Microwave transmission through a two-dimensional, isotropic, left-handed metamaterial," Appl. Phys. Lett., vol. 78, no. 4, 2001.

[12] E. Ubeda, A. Heldring, J. Parron, J. Romeu, and J. Rius, "Radiation pattern of composite finite arrays of conduting elements with an exciting elementary dipole," presented at the IEEE AP-Symp., Columbus, $\mathrm{OH}$, Jun. 22-27, 2003.

[13] S. M. Rao, D. R. Wilton, and A. W. Glisson, "Electromagnetic Scattering by Surfaces of Arbitrary Shape," IEEE Trans. Antennas Propag., vol. AP-30, no. 3, pp. 409-418, May 1982.

[14] D. R. Wilton, A. W. Glisson, D. H. Schaubert, O. M. Al-Bundak, and C. M. Butler, "Potential Integrals for uniform and linear source distributions on polygonal and polyhedral domains," IEEE Trans. Antennas Propag., vol. AP-32, no. 3, pp. 276-281, Mar. 1984.

[15] E. Ubeda and J. M. Rius, "Análisis eficiente de Slabs de Metamateriales," in Simposium Nacional de la Unión Científica internacional de Radio, Barcelona, Spain, Sep. 8-10, 2004.

[16] A. Heldring, J. M. Rius, L. P. Ligthart, and A. Cardama, "Accurate numerical modeling of the TARA reflector system," IEEE Trans. Antennas Propag., vol. 52, no. 7, pp. 1758-1766, Jul. 2004.

\section{Physical Insight Into the "Growing” Evanescent Fields of Double-Negative Metamaterial Lenses Using Their Circuit Equivalence}

\author{
Andrea Alù and Nader Engheta
}

Abstract-Pendry in his paper, "Negative refraction makes a perfect lens" (Phys. Rev. Lett., vol. 85, no. 18, pp. 3966-3969, 2000) put forward an idea for a lens made of a lossless metamaterial slab with $n=-1$, that may provide focusing with resolution beyond the conventional limit. In his analysis, the evanescent wave inside such a lossless double-negative (DNG) slab is "growing," and thus it "compensates" the decaying exponential outside of it, providing the subwavelength lensing properties of this system. Here, we examine this debated issue of "growing exponential" from an equivalent circuit viewpoint by analyzing a set of distributed-circuit elements representing evanescent wave interaction with a lossless slab of DNG medium. Our analysis shows that, under certain conditions, the current in series elements and the voltage at the element nodes may attain the dominant increasing due to the suitable resonance of the lossless circuit, providing an alternative physical explanation for "growing exponential" in Pendry's lens and similar subwavelength imaging systems.

Index Terms-Double-negative (DNG) metamaterials, left-handed (LH) metamaterials, subwavelength resolution.

\section{INTRODUCTION}

The idea of left-handed (LH) media, which dates back to 1967 when Veselago [1], theoretically studied plane wave propagation in materials in which he assumed both permittivity and permeability simultaneously having negative real parts, has attracted a great deal of attention in recent years. Various problems and ideas involving such media have been proposed and studied by many research groups. One such idea, namely a lens with possibility of perfect focusing, was theoretically suggested by Pendry in [2]. In his analysis, Pendry shows how evanescent waves, which are effectively responsible for subwavelength resolution, impinging on a suitably designed slab of double-negative (DNG) [3] material, may grow exponentially inside such a slab, and how this effect may "compensate" the decaying exponential taking place outside the slab [2]. This issue of "growing exponential" and subwavelength imaging has become the subject of interest for several research groups working in metamaterial research (see, e.g., [4]-[7]). Analogous subwavelength focusing and growing evanescent distributions have been demonstrated in two-dimensional negative-refractive-index transmission line structures [8], [9].

In one of our previous works, we have shown how a similar phenomenon of "growing exponential" may occur in pairs of "conjugate" metamaterial slabs, i.e., pairs of DNG and double-positive (DPS) slabs or pairs of single-negative (SNG) layers such as epsilon-negative (ENG) and $\mu$-negative (MNG) layers [10]. In these cases, we have shown how wave tunneling, transparency, and virtual image subwavelength displacement may be achieved under a proper choice of combinations

Manuscript received August 18, 2004; revised October 3, 2005. This work was supported in part by the Fields and Waves Laboratory, Department of Electrical and Systems Engineering, University of Pennsylvania. The work of A. Alù was supported by the scholarship "Isabella Sassi Bonadonna" from the Italian Electrical Association (AEI).

A. Alù is with the Department of Applied Electronics, University of Roma Tre, Rome, Italy and also with the Department of Electrical and Systems Engineering, University of Pennsylvania, Philadelphia, PA 19104 USA (e-mail: alu@uniroma3.it; andreaal@ee.upenn.edu).

N. Engheta is with the Department of Electrical and Systems Engineering, University of Pennsylvania, Philadelphia, PA 19104 USA (e-mail: engheta@ ee.upenn.edu.

Digital Object Identifier 10.1109/TAP.2005.861509 